\title{
Blood Glucose Measurement
}

National Cancer Institute

\section{Source}

National Cancer Institute. Blood Glucose Measurement. NCI Thesaurus. Code 692744.

A quantitative measurement for assessing the amount of glucose present in a blood sample. 